\title{
Size and Shape Dependence on Melting Temperature of Gallium Nitride Nanoparticles
}

\author{
Paneerselvam Antoniammal and Dakshanamoorthy Arivuoli \\ Crystal Growth Centre, Anna University, Chennai, Tamil Nadu 600 025, India \\ Correspondence should be addressed to Dakshanamoorthy Arivuoli, arivuoli@annauniv.edu \\ Received 19 January 2012; Revised 6 March 2012; Accepted 7 March 2012 \\ Academic Editor: Grégory Guisbiers
}

Copyright ( $) 2012$ P. Antoniammal and D. Arivuoli. This is an open access article distributed under the Creative Commons Attribution License, which permits unrestricted use, distribution, and reproduction in any medium, provided the original work is properly cited.

\begin{abstract}
The study of variation of the size and shape effect on the melting property of gallium nitride nanoparticles with their spherical and cylindrical geometrical feature is theoretically explored. A numerical thermodynamical model has been devoted for the study. A comparative investigation is made between the two shapes, at the range of $\sim 3 \mathrm{~nm}$ dia. The cylindrical GaN nanoparticles, whose melting point has been reported to decrease with decreasing particle radius, become larger than spherical-shaped nanoparticles. The melting temperature obtained in the present study is in line with the function of radius of curvature.
\end{abstract}

\section{Introduction}

It has been well established both experimentally and theoretically that the melting temperature $(T)$ of nanoparticles depends on the particle size [1-11]. Pawlow in 1909 developed a thermodynamical model that predicts a point depression of nanoparticles with the particle size. An attempt to confirm this experimentally has been made first by Pawlow [12] in 1910. Subsequently, other researchers have investigated the variation of melting temperature with particle size [13-17].

A numerical thermodynamical model is implemented for our exploration of the temperature distribution with respect to other size of the spherical and cylindrical nanoparticles of gallium nitride $(\mathrm{GaN})$. In the last decade, GaN has been investigated intensively, both experimentally and theoretically [18-22]. It is a wide bandgap semiconductor that exists in both wurtzite and zinc blende crystal structure. All devices, currently constructed for applications with the use of wurtzite structure. Because of the wide bandgap, the intrinsic carrier concentration $n_{i}$ of GaN is essentially Zero at $T=300 \mathrm{~K}$, and $n_{i}$ remains small enough so as to have a negligible effect on the operation of most of the devices until $T \approx 1000 \mathrm{~K}$. This property makes this wide bandgap material suitable for use in high-temperature environments. GaN is a very hard, mechanically stable wide bandgap semiconductor material with high heat capacity and thermal conductivity [18]. GaN can be doped with silicon (Si) or with oxygen to n-type and with magnesium $(\mathrm{Mg})$ to $\mathrm{p}$ type [19]; however, the $\mathrm{Si}$ and $\mathrm{Mg}$ atoms change the way GaN crystals grow, introducing tensile stresses and making them brittle [20]. GaN compounds also tend to have a high spatial defect frequency, on the order of a hundred million to ten billion defects per square centimeter. The crystalline quality GaN led to the discovery of p-type GaN [19], p-n junction blue/UV-LEDs [19], and room-temperaturestimulated emission. The very high breakdown voltage [21] high electron mobility, and saturation velocity of GaN has also made it an ideal candidate for high-power and hightemperature microwave applications, as evidenced by its high Johnson's figure of merit. Moreover, GaN-based MOSFET and MESFET transistors also offer many advantages in high-power electronics, especially in automotive and electric car applications [22]. Nanotubes of GaN are proposed for applications in nanoscale electronics, optoelectronics, and biochemical-sensing applications. They are also useful in military electronics such as active electronically scanned array radars. A GaN nanoparticle in a $\mathrm{BN}$ matrix by nitridation with urea has a lot of applications. Bionanotechnology is the use of biomolecules for applications in nanotechnology, including use of viruses. 
The interest has been heightened recently at the nanoscale, because nanostructures are pervasive in nature and in modern industry. We depict that the variation of the properties of GaN material with its geometrical feature size has a long history because of its importance in many fields. In physics and chemistry, the effect of particle size on melting has been discussed since 1900s, and this effect is not restricted to any particular material; rather, it is observed in a variety of materials from metals and alloys, and the typical size range over which the melting temperature undergoes a large change in the range 5-100 nm [23-31]. Many phenomena in solid-state physics and materials science also exhibit size dependence. For example, the elastic constants of $\mathrm{Ag}$ and $\mathrm{Pb}$ nanowires of diameter $30 \mathrm{~nm}$ are nearly twice those of the bulk metals [32]. Such increase in stiffness cannot be explained by structural modifications of the materials as the nanoscale reduction in the size of the solids also results in a change of their failure mode.

Thus, when the size of brittle calcium carbonate particles is reduced to a critical value of $850 \mathrm{~nm}[33,34]$, the particles behave as if they were ductile. Size dependence of the melting temperature at nanoscale has enormous implications in the production of nanocrystals and in the thermal stability of quantum dots. A large data has accumulated on this size dependence, and a number of theoretical models have been proposed to explain it. Thermodynamically, the melting temperature of nanoparticles has been described by three models: (1) the homogeneous melting and growth, (2) the liquid shell nucleation, and (3) the liquid nucleation and growth. All the three models predict a size-dependent melting temperature.

The melting temperature of the nanoparticles will be different in different shapes when considering the radius of curvature of nanoparticles; especially the particle size is mentioned with respect to its radius of curvature. Therefore, an indispensable course of action to take the particle size into consideration is when one develops the models for the melting temperature of nanoparticles. In the present work, the radius of curvature is considered to account for the particle shape difference and develop the model for the cylindrical nanoparticles. According to the relation between the melting temperature and radius of curvature of the nanoparticles, an expression (15) for the size- and shape-dependent melting temperature of nanoparticles is developed. The theoretical prediction of this expression for the melting temperature of $\mathrm{GaN}$ nanoparticles is compared between spherical and cylindrical shapes.

\section{Model of Calculation}

2.1. Surface Energy Calculation. It is known that the total surface energy involved in any heterogeneous nucleation is given as

total surface energy $=$ free surface energy

$$
\begin{aligned}
& \text { + interfacial energy } \\
& + \text { substrate energy. }
\end{aligned}
$$

It has been assumed that the geometry of the heterogeneous nucleation is that of the cylindrical-shaped particle. So the equation can be written as

$$
\Gamma=2 \pi R H \gamma_{s}+\pi H(2 R-H)\left(\gamma_{s b}-\gamma_{b}\right)+\Gamma_{b},
$$

where $R, H$ are the radius and the height of the cylindrical nanoparticle on the substrate, $\gamma_{s}$ is the surface energy of the solid vapor interface, $\gamma_{b}$ is the surface energy of the bare substrate, $\gamma_{s b}$ is the interfacial energy between the solid and the substrate and $\Gamma_{b}$ is the total energy of the substrate.

Now, (2) can be minimized by using the condition

$$
\frac{\partial \Gamma}{\partial R}+\frac{\partial \Gamma}{\partial H}=0
$$

To find the relationship between $R$ and $H$, substituting (2) in (3), we get

$$
H=\frac{\Delta \gamma_{s b}}{\gamma_{s}} R,
$$

where $\Delta \gamma_{s b}$ is a parameter called wetting or spreading parameter which is given by

$$
\Delta \gamma_{s b}=\gamma_{b}-\gamma_{s}-\gamma_{s b}
$$

The magnitude of the wetting or spreading parameter $\left(\Delta \gamma_{s b}\right)$ is used to determine whether the surface melting takes place or not in the given substrate. If the magnitude of the spreading parameter is positive, that is, $\Delta \gamma_{s b}>0$, then there is a possibility of wetting the substrate, and if it is negative, that is, $\Delta \gamma_{s b}<0$, then the surface melting takes place.

In the case of nanofilms, the free surface energy of the solid is always higher than the energy density of the bare substrate, thus, $\Delta \gamma_{s b}$ is always less than zero and it is negative. From (5), it is seen clearly that if $\Delta \gamma_{s b}$ is negative, then $H$ is also negative, and this is not possible. Thus, to keep $H$ positive, a negative sign is included in (4), that is,

$$
H=-\frac{\Delta \gamma_{s b}}{\gamma_{s}} R
$$

Substituting the value of $H$ (height of the spherical nanoparticle on the substrate) in (3), we get

$$
\Gamma^{*}=2 \pi \gamma_{s}\left(\frac{a}{R_{s}}\right) a^{2}+\Gamma_{b} .
$$

$R_{s}^{*}$ is the corresponding radius of curvature of supported spherical nanoparticle, and it is given by

$$
R_{s}^{*}=\left[\frac{1}{2}\right]^{1 / 3}\left[\frac{2 \gamma_{s}}{\Delta \gamma_{s b}}\right]^{2 / 3} \frac{a}{\left[3+\Delta \gamma_{s b} / \gamma_{s}\right]^{1 / 3}},
$$

where $\Gamma^{*}$ is the equilibrium surface energy, and $R_{s}^{*}$ is the corresponding radius of curvature of the supported gallium 
nitride $(\mathrm{GaN})$ solid spherical nanoparticles [35] and it is given by

$$
R_{s}^{*}=L_{G}\left(1-\frac{\delta\left(4 \gamma_{s}^{2} \pi^{2}\right)^{3}}{\left[r\left(4 \gamma_{s}^{2} \pi^{2}\right)+\left(\gamma_{b}-\gamma_{s}-\gamma_{s b}\right)^{2}\right]^{3}}\right),
$$

where $L_{G}$ is latent heat of melting material of GaN, $\delta$ is fitting parameter, and it is a measure of GaN solid shell.

Similarly, the corresponding radius of curvature of the GaN nanoparticle with liquid film surrounding the solid [35] $R_{l}^{*}$ is given as

$$
R_{l}^{*}=L_{G}\left(1-\frac{\delta\left(4 \gamma_{l}^{2} \pi^{2}\right)^{3}}{\left[r\left(4 \gamma_{l}^{2} \pi^{2}\right)+\left(\gamma_{b}-\gamma_{l}-\gamma_{l b}\right)^{2}\right]^{3}}\right),
$$

where $\gamma l$ is the free surface energy of the liquid-vapors interface, and $\gamma_{l b}$ is the interfacial energy between the liquid and substrate. The basic formula for the radius of curvature of cylindrical nanoparticle which is used in the above expression has been attached in the Appendix [35].

2.2. Melting Point Calculation. The expression for the melting of nanoparticle can be derived using free energies density of solid and liquid. The free energy density expression for the solid $\left(F_{s}\right)$ and the corresponding liquid $(F l)$ are given as follows [36]:

$$
\begin{aligned}
& F_{s}=\frac{4}{3} \pi a^{3} \rho f_{s}+4 \pi a^{3} \rho \frac{\gamma_{s}}{R_{s}^{*}}, \\
& F_{l}=\frac{4}{3} \pi a^{3} \rho f_{l}+4 \pi a^{3} \rho \frac{\gamma_{l}}{R_{l}^{*}},
\end{aligned}
$$

where $a$ is the radius of the melting of nanoparticle, $f_{s}$ is the surface energy of solid, and $f_{l}$ is the surface energy of liquid. Now the difference in free energies $(\Delta F)$ is calculated to be

$$
\begin{gathered}
\Delta F=F_{s}-F_{l}, \\
\Delta F=\frac{4}{3} \pi a^{3} \rho\left[\left(f_{s}-f_{l}\right)+3\left(\frac{\gamma_{s}}{R_{s}^{*}}-\frac{\gamma_{l}}{R_{l}^{*}}\right)\right] .
\end{gathered}
$$

It is already known that

$$
f_{l}-f_{s}=\rho L\left(1-\frac{T}{T_{c}}\right),
$$

where $\rho$ is the density of the materials, $L$ is the latent heat of the material, $T_{c}$ is the bulk melting temperature, and $T$ is the melting point of the nanomaterial as a function of size. At melting point the difference in free energies is zero, that is, $\Delta F=0$. Substituting the above two equations, we get

$$
T_{m}=T_{c}\left(1-\frac{2}{\rho L}\left(\frac{\gamma_{s}}{R_{s}^{*}}-\frac{\gamma_{l}}{R_{l}^{*}}\right)\right) .
$$

If $R_{s}^{*}=R_{l}^{*}=R^{*}$, that is, if no surface melting takes place then, $T_{m}=T_{m}^{\text {free }}\left(R^{*}\right)$. $T_{m}^{\text {free }}$ is the melting point of nanofilms with free interfacial energy between liquid and substrate.

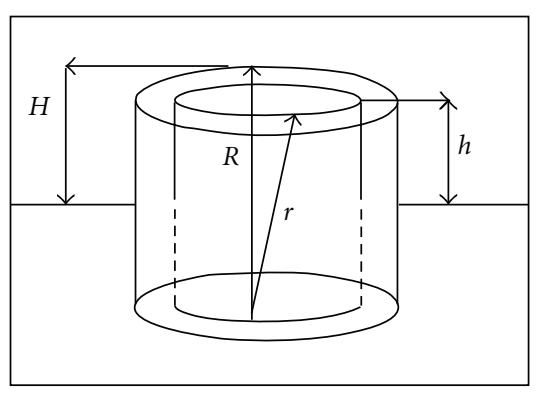

Figure 1: $H$ : height of cylindrical particle with quasiliquid film. $R$ : radius of curvature of quasi liquid film formed on the core cylindrical solid particle. $r$ : radius of curvature of the core cylindrical solid particle. $h$ : height of the core cylindrical solid particle.

Thus, the expression for melting point for nanofilms $\left(T_{m}\right)$ as a function of radius of the particle is given as follows:

$$
T_{m}=T_{m}^{\text {free }}\left(1-\frac{2}{\rho L}\left(\frac{\gamma_{s}}{R_{s}^{*}}-\frac{\gamma_{l}}{R_{l}^{*}}\right)\right) .
$$

Using the expression above, the variation of the melting point with respect to the $\mathrm{GaN}$ particle can be plotted.

Similarly, the expression for the melting point GaN nanofilms as a function of the radius of the particle [35] is given as

$$
T_{\mathrm{GN}}=T\left[1-\frac{2}{\rho_{s} L_{G}}\left(\frac{\gamma_{s}}{R_{s}}-\frac{\gamma_{l}}{R_{l}}\left(\frac{\rho_{s}}{\rho_{l}}\right)^{2 / 3}\right)\right],
$$

where $T_{\mathrm{GN}}$ is the temperature of nanoparticle of $\mathrm{GaN}, T$ is the Bulk melting temperature of GaN, $\rho_{s}$ Density of GaN solid phase, $\rho_{l}$ is the density of GaN liquid phase, $L_{G}$ is the latent heat of melting $\mathrm{GaN}$ material, $\gamma_{s}$ is the surface energy of solid GaN, $\gamma_{l}$ is the surface energy of liquid $\mathrm{GaN}, R_{s}$ is the radius of curvature of supported $\mathrm{GaN}$ nanoparticle, and $R_{l}$ is the radius of curvature of nanoparticle with liquid film.

2.3. Surface Melting Calculations. The schematic diagram for the surface melting is as shown in Figure 1. A quasiliquid film of radius $R$ is formed on the core solid particle of radius $r$. The difference between $R$ and $r$ gives the thickness of the liquid film.

At the onset of surface melting, we assume that the geometry is close to that of the solid particle in a quasiliquid film of radius of curvature $R$ formed on the core solid particle of radius of curvature $r$. The difference between $R$ and $r$ gives the thickness of the liquid film. The solid particle is in its equilibrium geometry and it is initially wet by a molten layer with uniform thickness. The radius of curvature of solid particle is $r$ and height is $h$ [35].

Liquid film thickness is given as

$$
\delta=H-h=R-r .
$$


The total energy of the surface-melted particle is then a function of $\delta$ and is given as

$$
F(\delta)=V_{s}(\delta)\left(f_{s}-f_{l}\right)+V_{l} f_{l}+\Gamma(\delta),
$$

where $V_{s}$ is the volume of the solid, $V_{l}$ is the volume of the liquid, $\Gamma(\delta)$ is the thickness-dependent surface energy.

\section{Results and Discussion}

The crystal shape and the supporting substrate influence the size dependence of melting point, where the cylindrical shape is considered. The mentioned gallium nitride $(\mathrm{GaN})$ nanoparticles are extremely small in $1-100 \mathrm{~nm}$ range. There is a considerable difference in the calculated values of the melting point as a function of size of the particle. It is known that the radius of curvature varies according to the shapes of the nanoparticle which decide the size of the particle.

The main difference between (8) and other expression (9) for the size-dependent melting temperature is that the radius of curvature of spherical and cylindrical shape is considered in (8) and (9), respectively, and derived using a separate helical method which is given in the Appendix. Based on expression (8), corresponding melting point for $\mathrm{GaN}$ is obtained in expression (15).

Using the expressions (8) and (15), the size of the supported spherical nanoparticle is calculated and also the variation of the melting point, which is listed in Table 1.

Similarly, using the expressions (9) and (16), the size of the supported cylindrical nanoparticle is calculated and also the variation of the melting point, which is listed in Table 2. The thickness of the quasiliquid film is plotted as a function of the size of the supported nanoparticles.

3.1. Melting Point versus Radius of the Particle. Using the data for the gallium nitride $(\mathrm{GaN})$ nanofilms in the expression that is derived for melting point, the variation of the melting point with respect to the size of the supported nanoparticles can be tabulated. Using Tables 1 and 2, the melting point can be plotted against the radius of the supported spherical and cylindrical nanoparticles, respectively.

From Figures 2 and 3, it can be clearly seen that with decrease in size of the supported nanoparticles, the melting point reduces. It is also seen that there is a variation in the melting point for free nanoparticles and the nanofilms. Thus, the effect of substrate is determined.

An important part of our present work is a comparative study of GaN film between spherical and cylindrical nanoparticles has been done, and the theoretical calculated values are tabulated in Table 3, comparative Figure 4 plotted by the tabulated values shows the variation of melting point of $\mathrm{GaN}$ nanofilms against the size of the spherical and cylindrical nanoparticles.

In this, it is clearly shown that the melting temperature decreases when the particle size reduces little more in the case of cylindrical than the spherical nanoparticle. At nanoscales, particles exhibit many thermophysical features distinct from those found at microscales. As the size decreases beyond

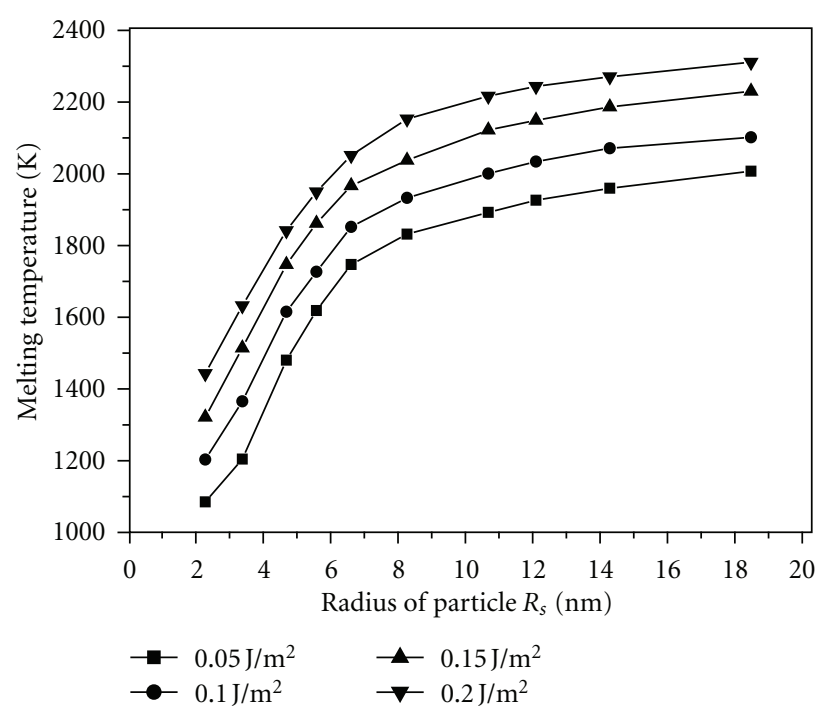

FIGURE 2: Variation of melting point of GaN spherical nanoparticles against the size of the particles.

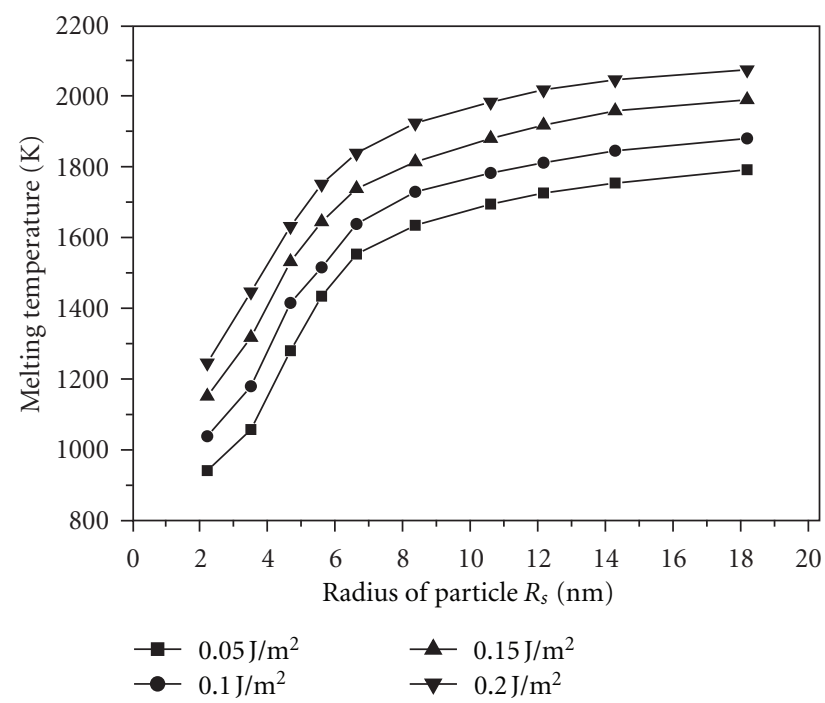

FIGURE 3: Variation of melting point of GaN cylindrical nanoparticles against the size of the particles.

a critical value, due to the increase in the surface-tovolume ratio, the melting temperature derivates from the bulk values and becomes a size-dependent property. This phenomenon has been studied experimentally by means of transmission electron diffraction by Wronski for nanosized tin [37]. The melting point agrees reasonably well with the predictions based on classical theories, which show a nonlinear relationship with the reciprocal of the particle size. The melting temperature of gallium nitride $(\mathrm{GaN})$ becomes size dependent for particles smaller than $10 \mathrm{~nm}$, reducing from $1618 \mathrm{~K}$ range for a $5 \mathrm{~nm}$ particle.

Pawlow [1] improved the Gibbs-Thompson model by considering the equilibrium of a liquid spherical drop with both a solid spherical particle of the same material and 
TABLE 1: Variations in melting point of GaN spherical nanoparticles against the size of the particles.

\begin{tabular}{|c|c|c|c|c|c|c|}
\hline \multirow{2}{*}{ S. no. } & \multirow{2}{*}{$R_{s}(\mathrm{~nm})$} & \multirow{2}{*}{$1 / R_{s}(\mathrm{~nm})^{-1}$} & \multicolumn{4}{|c|}{$T_{\mathrm{GN}}(\mathrm{K})$} \\
\hline & & & $\gamma_{l b}=0.05 \mathrm{~J} / \mathrm{m}^{2}$ & $\gamma_{l b}=0.10 \mathrm{~J} / \mathrm{m}^{2}$ & $\gamma_{l b}=0.15 \mathrm{~J} / \mathrm{m}^{2}$ & $\gamma_{l b}=0.20 \mathrm{~J} / \mathrm{m}^{2}$ \\
\hline 1 & 2.28 & 0.439 & 1085.07 & 1203.18 & 1321.29 & 1443.12 \\
\hline 2 & 3.38 & 0.296 & 1204.39 & 1365.48 & 1514.15 & 1632.27 \\
\hline 3 & 4.69 & 0.213 & 1480.29 & 1615.33 & 1747.07 & 1841.65 \\
\hline 4 & 5.58 & 0.179 & 1618.64 & 1726.84 & 1861.88 & 1949.85 \\
\hline 5 & 6.61 & 0.151 & 1747.07 & 1851.97 & 1966.78 & 2051.33 \\
\hline 6 & 8.27 & 0.121 & 1831.74 & 1932.92 & 2037.81 & 2152.92 \\
\hline 7 & 10.68 & 0.094 & 1892.44 & 2000.64 & 2122.06 & 2216.63 \\
\hline 8 & 12.10 & 0.083 & 1926.31 & 2034.11 & 2149.32 & 2243.89 \\
\hline 9 & 14.29 & 0.069 & 1959.76 & 2071.26 & 2186.48 & 2270.73 \\
\hline 10 & 18.48 & 0.054 & 2007.25 & 2101.82 & 2230.26 & 2311.21 \\
\hline
\end{tabular}

TABLE 2: Variations in melting point of GaN cylindrical nanoparticles against the size of the particles.

\begin{tabular}{|c|c|c|c|c|c|c|}
\hline \multirow{2}{*}{ S. no. } & \multirow{2}{*}{$R_{s}(\mathrm{~nm})$} & \multirow{2}{*}{$1 / R_{s}(\mathrm{~nm})^{-1}$} & \multicolumn{4}{|c|}{$T_{\mathrm{GN}}(\mathrm{K})$} \\
\hline & & & $\gamma_{l b}=0.05 \mathrm{~J} / \mathrm{m}^{2}$ & $\gamma_{l b}=0.10 \mathrm{~J} / \mathrm{m}^{2}$ & $\gamma_{l b}=0.15 \mathrm{~J} / \mathrm{m}^{2}$ & $\gamma_{l b}=0.20 \mathrm{~J} / \mathrm{m}^{2}$ \\
\hline 1 & 2.23 & 0.448 & 941.41 & 1038.43 & 1151.56 & 1245.51 \\
\hline 2 & 3.52 & 0.284 & 1057.22 & 1179.55 & 1317.61 & 1446.46 \\
\hline 3 & 4.69 & 0.213 & 1280.02 & 1415.01 & 1531.21 & 1631.29 \\
\hline 4 & 5.61 & 0.178 & 1433.81 & 1515.48 & 1643.95 & 1750.56 \\
\hline 5 & 6.64 & 0.151 & 1553.06 & 1637.81 & 1737.91 & 1838.37 \\
\hline 6 & 8.38 & 0.118 & 1634.36 & 1728.71 & 1813.45 & 1923.12 \\
\hline 7 & 10.61 & 0.094 & 1694.18 & 1782.01 & 1879.41 & 1982.56 \\
\hline 8 & 12.17 & 0.082 & 1725.63 & 1810.98 & 1916.99 & 2017.07 \\
\hline 9 & 14.29 & 0.071 & 1753.62 & 1844.89 & 1957.64 & 2045.45 \\
\hline 10 & 18.19 & 0.055 & 1791.21 & 1879.41 & 1989.08 & 2073.83 \\
\hline
\end{tabular}

its vapor. This model leads to the following Well-Lenon Equation (15). The suggestion that the melting point of a particle should depend on its size is implicit in the work Thomson [38].

It is well known that the melting temperature of $\mathrm{Au}$ $(1064 \mathrm{~K})$ decreases when particle dimensions are reduced to the nanoscale. Therefore, at $\sim 3 \mathrm{~nm}$ diameter, Au particle can melt at temperature $\sim 500 \mathrm{~K}[23,39,40]$. Similarly, the melting temperature of $\mathrm{B}_{4} \mathrm{C}(2450 \mathrm{~K})$ lowered to $\sim 764 \mathrm{~K}$ range with spherical-shaped and $\sim 495 \mathrm{~K}$ ranges with cylindrical shaped nanoparticles at $\sim 3 \mathrm{~nm}$ dia [35]. In the present work, the melting temperature of GaN (2770 K) decreases significantly when the particle dimensions are reduced to the nanoscale and we got that an $\sim 3 \mathrm{~nm}$ diameter of GaN spherical shaped particle melted at temperature $\sim 1747 \mathrm{~K}$ range, but the same particle with an $\sim 3 \mathrm{~nm}$ diameter melted at the temperature $\sim 1553 \mathrm{~K}$ range, since the particle shape is considered in cylindrical shape.

3.2. Melting Point versus Inverse of the Radius of the Particle. In Figures 5 and 6 there are two regions namely, logarithmic increases at low values of radius and an exponential increase at the higher values of radius. A linear figure (Figures 5 and 6) between melting point and the inverse of the radius of the particle is drawn.

3.3. Thickness of the Liquid Film versus Radius of the Particle. From Figure 2 it can be seen clearly that with increased in the radius of the supported nanoparticles, it increases up to certain limit after which it is saturated. The melting temperature is a function of the particle radius evaluated from the calculations and the analytical formulation reported in Buffat and Borel [23]. In that, the calculation of latent heat is a function of the particle radius, which is valid for small particle with spherical shape, and it is the radius of curvature varies according to the shape of the particle. The obtained expression (9) because is for the corresponding radius of curvature of the GaN solid cylindrical nanoparticle. Similarly, the expression (16) obtained for the melting point of GaN nanofilms is a function of radius of the particle.

Melting-point depression is most evident in nanowires, nanotubes, and nanoparticles, which all melt at lower temperatures than bulk amounts of the same material. Changes in melting point occur because nanoscale materials have a much larger surface-to-volume ratio than bulk materials, drastically altering their thermodynamic and thermal properties. The decrease in melting temperature can be on 
TABLE 3: Comparative study of GaN film between spherical and cylindrical nanoparticle.

\begin{tabular}{|c|c|c|c|c|c|c|c|c|c|c|}
\hline \multirow{3}{*}{ S. no. } & \multirow{2}{*}{\multicolumn{2}{|c|}{$R_{s}(\mathrm{~nm})$}} & \multicolumn{8}{|c|}{$T_{m}(\mathrm{~K})$} \\
\hline & & & \multicolumn{2}{|c|}{$\gamma_{l b}=0.05 \mathrm{~J} / \mathrm{m}^{2}$} & \multicolumn{2}{|c|}{$\gamma_{l b}=0.10 \mathrm{~J} / \mathrm{m}^{2}$} & \multicolumn{2}{|c|}{$\gamma_{l b}=0.15 \mathrm{~J} / \mathrm{m}^{2}$} & \multicolumn{2}{|c|}{$\gamma_{l b}=0.20 \mathrm{~J} / \mathrm{m}^{2}$} \\
\hline & S & $\mathrm{C}$ & S & $\mathrm{C}$ & S & $\mathrm{C}$ & S & $\mathrm{C}$ & S & $\mathrm{C}$ \\
\hline 1 & 2.28 & 2.23 & 1085.07 & 941.41 & 1203.18 & 1038.43 & 1321.29 & 1151.56 & 1443.12 & 1245.51 \\
\hline 2 & 3.38 & 3.52 & 1204.39 & 1057.22 & 1365.48 & 1179.55 & 1514.15 & 1317.61 & 1632.27 & 1446.46 \\
\hline 3 & 4.69 & 4. 69 & 1480.29 & 1280.02 & 1615.33 & 1415.01 & 1747.07 & 1531.21 & 1841.65 & 1631.29 \\
\hline 4 & 5.58 & 5.61 & 1618.64 & 1433.81 & 1726.84 & 1515.48 & 1861.88 & 1643.95 & 1949.85 & 1750.56 \\
\hline 5 & 6.61 & 6.64 & 1747.07 & 1553.06 & 1851.97 & 1637.81 & 1966.78 & 1737.91 & 2051.33 & 1838.37 \\
\hline 6 & 8.27 & 8.38 & 1831.70 & 1634.36 & 1932.92 & 1728.71 & 2037.81 & 1813.45 & 2152.92 & 1923.12 \\
\hline 7 & 10.68 & 10.61 & 1892.44 & 1694.18 & 2000.64 & 1782.01 & 2122.06 & 1879.41 & 2216.63 & 1982.56 \\
\hline 8 & 12.10 & 12.17 & 1926.31 & 1725.63 & 2034.11 & 1810.98 & 2149.32 & 1916.99 & 2243.89 & 2017.07 \\
\hline 9 & 14.29 & 14.29 & 1959.76 & 1753.62 & 2071.26 & 1844.89 & 2186.48 & 1957.64 & 2270.73 & 2045.45 \\
\hline 10 & 18.48 & 18.19 & 2007.25 & 1791.21 & 2101.82 & 1879.41 & 2230.26 & 1989.08 & 2311.21 & 2073.83 \\
\hline
\end{tabular}

Note: C: cylindrical Nanoparticle/S: spherical Nanoparticle.

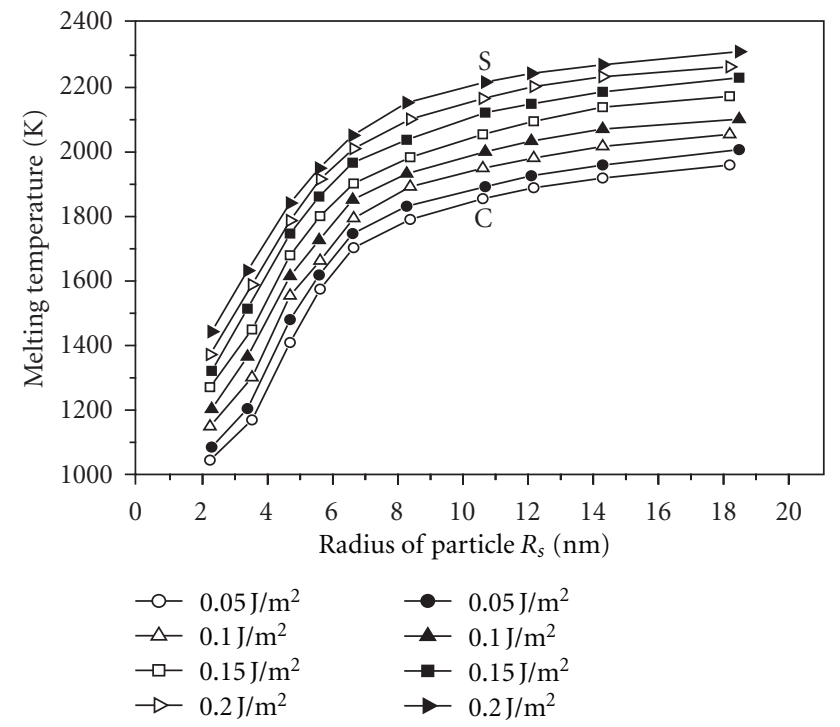

Figure 4: Comparative study of GaN film between spherical and cylindrical nanoparticles.

the order of tens to hundreds of degrees for metals with nanometer dimensions [41-44]. Surface atoms bind in the solid phase with less cohesive energy because they have fewer neighboring atoms in close proximity compared to atoms in the bulk of the solid. Each chemical bond an atom shares with a neighboring atom provides cohesive energy, so atoms with fewer bonds and neighboring atoms have lower cohesive energy. The average cohesive energy per atom of a nanoparticle has been theoretically calculated as a function of particle size [45].

Atoms located at or near the surface of the nanoparticle have reduced cohesive energy due to a reduced number of cohesive bonds. An atom experiences an attractive force with all nearby atoms according to the Lennard-Jones potentials [46]. The cohesive energy of an atom is directly related to

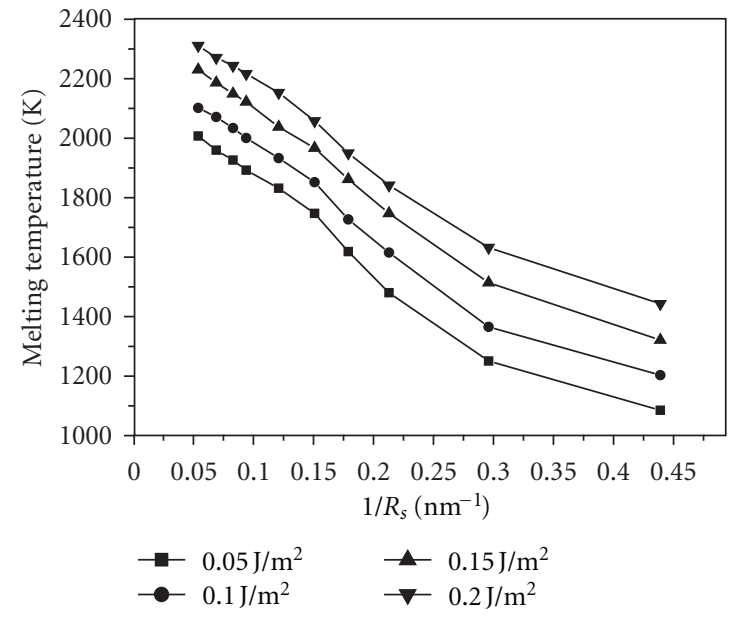

FIGURE 5: Variation of the melting point of GaN with inverse of radius of the particle in the spherical shape.

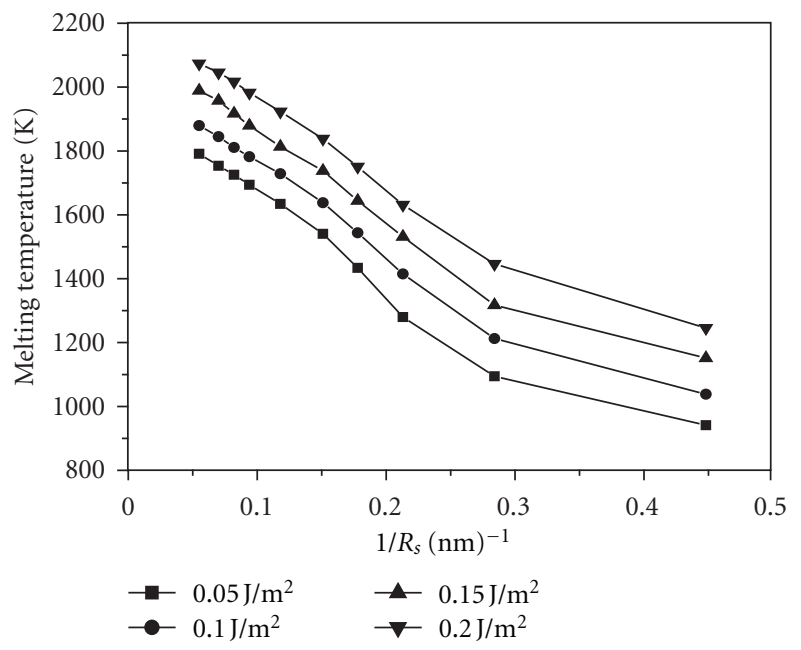

FIGURE 6: Variation of the melting point of GaN with inverse of radius of the particle in the cylindrical shape. 
the thermal energy required to free the atom from the solid. Since atoms near the surface have fewer bonds and reduced cohesive energy, they require less energy to be free from the solid phase. Melting point depression of high surfaceto-volume ratio materials results from this effect [47]. The liquid drop model (LDM) represents the binding energy of a nanoparticle as a function of the free energies of the volume and surface [46]. The liquid shell nucleation model (LSN) predicts that a surface layer of atoms melts prior to the bulk of the particle [48]. The melting temperature of a nanoparticle is a function of its radius of curvature according to the LSN. The bond-order-length-strength (BOLS) model calculates the melting temperature for individual atoms from the sum of their cohesive bonds. As a result, the BOLS predicts the surface layers of a nanoparticle melt at lower temperatures than the bulk of the nanoparticle [49]. The lowered coordination number changes the equilibrium bond length between atoms near the surface of the nanoparticle. The integrated cohesive energy for surface atoms is much lower than bulk atoms due to the reduced coordination number and overall decrease in cohesive energy.

Nanoparticle shape impacts the melting point of a nanoparticle. Facets, edges, and deviations from a perfect sphere all change the magnitude of melting point depression [46]. These shape changes affect the surface-to-volume ratio, which affects the cohesive energy and thermal properties of a nanostructure. The cohesive energy of nanocrystals, which can be determined by experiment [50] and computed by different theoretical models such as the SE model [51], the BE model [52], the Lennard-Jones potential model [53], Jiang's model [54], the liquid-drop model [55-57], and the bondOLS model $[58,59]$, is regarded as directly related to the nature of the thermal stability of nanocrystals [60].

In observing the evolution of numerical thermodynamical model yields the calculated data, which is compared to the melting point of spherical nanoparticle, the cylindrical nanoparticle has low melting point, since the surface area of cylindrical nanoparticle $\left(169.56 \mathrm{~nm}^{2}\right.$ at $\sim 3 \mathrm{~nm}$ radius range) is larger than the spherical $\left(113.04 \mathrm{~nm}^{2}\right.$ at $\sim 3 \mathrm{~nm}$ radius range). The number of atoms present on the surface will have only fewer neighboring atoms. Hence, on the larger surface, the more number of atoms will have fewer neighboring atoms in close proximity compared to atoms in the bulk of the nanomaterial. Each chemical bond of an atom shared with a neighboring atom provides cohesive energy, so atom with fewer bonds and neighboring atoms have lower cohesive energy; they require less energy to be free from the solid phase. Hence, melting point depression of high surface (cylindrical shape) is reduced more than the lower surface (spherical shape) nanoparticles. It should be mentioned that the radius of curvature with respect to the shape of the nanoparticle and as the present work describes the melting temperature effect on the shape difference between the spherical nanoparticles and cylindrical nanoparticles. This same property of GaN nanowires with triangular cross-section has been studied using molecular dynamics simulation, in that it was reported that the melting temperature of the $\mathrm{GaN}$ nanowires increases with increasing cross-sectional area to a saturation value [61]. The "approximately" is

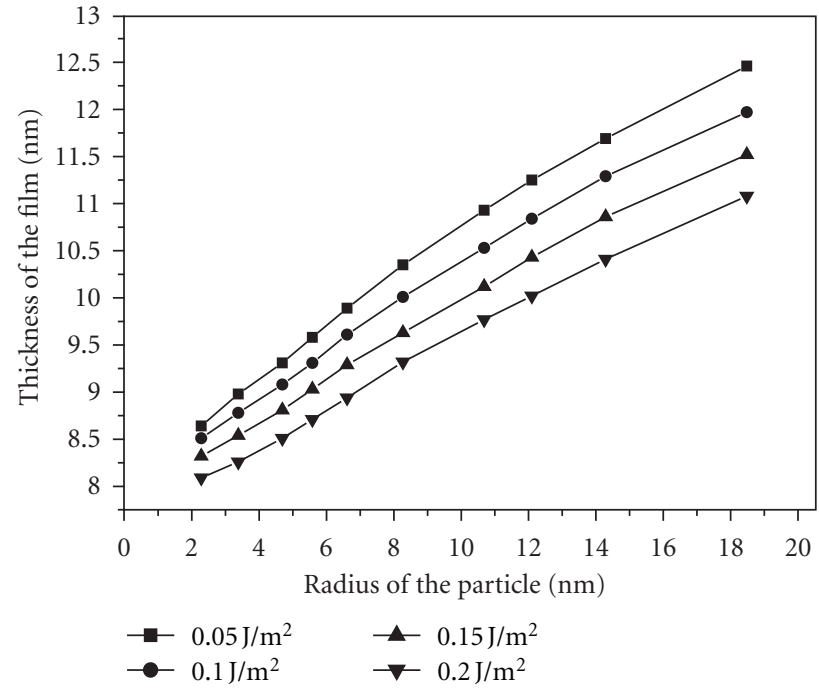

FIGURE 7: Variation of the thickness of the liquid film against radius of $\mathrm{GaN}$ spherical particles.

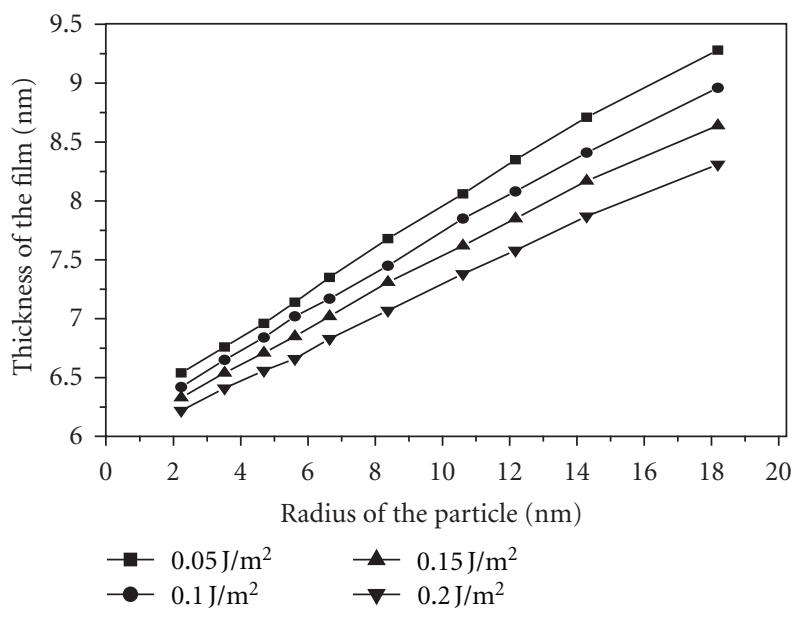

FIGURE 8: Variation of the thickness of the liquid film against radius of GaN cylindrical particles.

stressed here due to the fact that some different shape of the nanoparticle may have different radius of curvature which decides the particle size. However, present calculation shows that the present radius of curvature expression is enough for predicting the shape-dependent size effect on the temperature of nanoparticle. This method could be used to study the nanoparticles, and it can be experimentally determined by measuring the particle shape with radius of curvature, which is subjected to future experiments.

It is reported that the atomic radius of metallic nanoparticles contracts with decreasing their particle size, which means that the atomic radius will change a little if the particle size is small. The thickness of the liquid film against the radius of the supported nanoparticle is also done for $\mathrm{GaN}$ which has been given in the tabulation (Tables 4 and 5) and in Figures 7 and 8. 
TABLE 4: The thickness of the liquid films is tabulated against the radius of the supported spherical nanoparticles.

\begin{tabular}{|c|c|c|c|c|c|}
\hline \multirow{2}{*}{ S. no. } & \multirow{2}{*}{$R_{s}(\mathrm{~nm})$} & \multicolumn{4}{|c|}{$\delta(\mathrm{nm})$} \\
\hline & & $\gamma_{l b}=0.05 \mathrm{~J} / \mathrm{m}^{2}$ & $\gamma_{l b}=0.10 \mathrm{~J} / \mathrm{m}^{2}$ & $\gamma_{l b}=0.15 \mathrm{~J} / \mathrm{m}^{2}$ & $\gamma_{l b}=0.20 \mathrm{~J} / \mathrm{m}^{2}$ \\
\hline 1 & 2.28 & 8.64 & 8.51 & 8.32 & 8.09 \\
\hline 2 & 3.38 & 8.98 & 8.78 & 8.54 & 8.26 \\
\hline 3 & 4.69 & 9.31 & 9.08 & 8.81 & 8.51 \\
\hline 4 & 5.58 & 9.58 & 9.31 & 9.03 & 8.71 \\
\hline 5 & 6.61 & 9.89 & 9.61 & 9.29 & 8.94 \\
\hline 6 & 8.27 & 10.35 & 10.01 & 9.63 & 9.32 \\
\hline 7 & 10.68 & 10.93 & 10.53 & 10.12 & 9.77 \\
\hline 8 & 12.10 & 11.25 & 10.84 & 10.43 & 10.02 \\
\hline 9 & 14.29 & 11.69 & 11.29 & 10.86 & 10.41 \\
\hline 10 & 18.48 & 12.46 & 11.97 & 11.52 & 11.08 \\
\hline
\end{tabular}

TABLE 5: The thickness of the liquid films is tabulated against the radius of the supported cylindrical nanoparticles.

\begin{tabular}{|c|c|c|c|c|c|}
\hline \multirow{2}{*}{ S. no. } & \multirow{2}{*}{$R_{s}(\mathrm{~nm})$} & \multicolumn{4}{|c|}{$\delta(\mathrm{nm})$} \\
\hline & & $\gamma_{l b}=0.05 \mathrm{~J} / \mathrm{m}^{2}$ & $\gamma_{l b}=0.10 \mathrm{~J} / \mathrm{m}^{2}$ & $\gamma_{l b}=0.15 \mathrm{~J} / \mathrm{m}^{2}$ & $\gamma_{l b}=0.20 \mathrm{~J} / \mathrm{m}^{2}$ \\
\hline 1 & 2.23 & 6.54 & 6.42 & 6.33 & 6.22 \\
\hline 2 & 3.52 & 6.76 & 6.65 & 6.54 & 6.41 \\
\hline 3 & 4.69 & 6.96 & 6.84 & 6.71 & 6.56 \\
\hline 4 & 5.61 & 7.14 & 7.02 & 6.85 & 6.66 \\
\hline 5 & 6.64 & 7.35 & 7.17 & 7.02 & 6.83 \\
\hline 6 & 8.38 & 7.68 & 7.45 & 7.31 & 7.07 \\
\hline 7 & 10.61 & 8.06 & 7.85 & 7.62 & 7.38 \\
\hline 8 & 12.17 & 8.35 & 8.08 & 7.85 & 7.58 \\
\hline 9 & 14.29 & 8.71 & 8.41 & 8.17 & 7.87 \\
\hline 10 & 18.19 & 9.28 & 8.96 & 8.64 & 8.31 \\
\hline
\end{tabular}

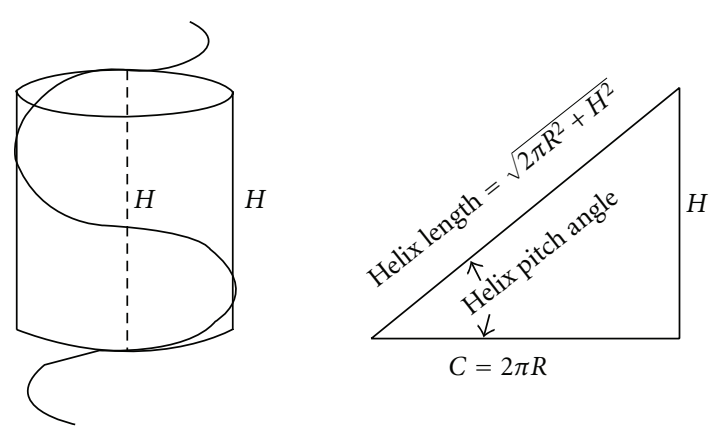

Figure 9

The regulation of nanotechnology has made a growing debate related to the human health and safety risks associated with nanotechnology [62]. An important property of the material, the melting temperature of $\mathrm{GaN}$, was predicted by top-down approach through classical thermodynamics [63]. Hence, the phenomenon of size and shape effect on melting temperature of nanoparticles is utilized in nanotechnology field. The expression for the size-dependent melting temperature of nanoparticles in present work is derived from their size-dependent radius of curvature according to the shape of the nanoparticles. It should be mentioned that, according to the shape of the nanoparticles, the radius of curvature varies. And it should be noted that the particle size-dependent on its radius of curvature. Similar melting phenomena have been reported for $\mathrm{Au}$ nanoparticles [19], and the melting temperature of prism-shaped nanoparticles [26] and especially for B4C nanoparticles [45] had been analyzed. It has been argued that substrate-particle interaction plays a significant role in the melting behavior of nanoparticles. It has also been shown that the extrapolated bulk melting temperature is lower than the experimental value. It has also been noted by different researchers that the bulk melting temperature cannot be extrapolated from the nanoscale results [64]. In our present work, we showed that the melting temperature of GaN decreased when the size of the nanoparticle reduced. Moreover this reduction is little more in cylindrical nanoparticle when compared with spherical nanoparticle.

\section{Conclusion}

The present exploration showed, the size- and shapedependent temperature of $\mathrm{GaN}$ nanoparticles, using the numerical thermodynamical model, where spherical and cylindrical shapes of the nanoparticle are considered with respect to its radius of curvature. It is shown that the present 
results of the melting temperature of $\mathrm{GaN}$ nanoparticles consistently decrease with the size of the nanoparticle. The cylindrical $\mathrm{GaN}$ nanoparticles, whose melting point has been decreased with decreasing particle radius, become larger than spherical-shaped nanoparticle. Further, it is found that the particle shape can affect the melting temperature of nanoparticles, and this effect on the melting temperature becomes larger with decreasing particle size. Because melting temperature is a very important parameter, the model developed in the present investigation may have potential applications in the temperature-related phenomena of nanoparticles.

\section{Appendix}

The following formula for radius of curvature of a cylinder (by Helix method) (see Figure 9):

$$
\begin{gathered}
\text { Helix length }=c \times \frac{c}{\text { helix length }}, \\
C=\text { helix length } \times \frac{\text { helix length }}{c}=2 \pi R^{*}, \\
R^{*}=\frac{\sqrt{\left(2 \pi R^{*}\right)^{2}+H^{2} \times \sqrt{(2 \pi R)^{2}+H^{2}}}}{2 \pi \times 2 \pi R}, \\
2 \pi R^{*}=\sqrt{\left(2 \pi R^{*}\right)^{2}+H^{2}} \times \sqrt{\frac{(2 \pi R)^{2}+H^{2}}{2 \pi R}}, \\
R^{*}=\frac{(\pi / 2)^{2} R^{2}+(H / 4)^{2}}{(\pi / 2)^{2} R},
\end{gathered}
$$

where $H$ : Height required for helix to complete one revolution about the cylinder, $c$ : Circumference of cylinder, $R$ : Radius of cylinder, $C$ : Circumference of baluster circle, $R^{*}$ : Radius of curvature of cylinder.

By using the above expression, the radius of curvature of solid cylindrical nanoparticle is obtained as

$$
R_{s}^{*}=\frac{R\left[\left(4 \gamma_{s}^{2} \pi^{2}\right)+\left(\gamma_{b}-\gamma_{s}-\gamma_{s b}\right)^{2}\right]}{4 \gamma_{s}^{2} \pi^{2}} .
$$

Similarly, the corresponding radius of curvature of the nanoparticle with a liquid film surrounding the solid is calculated to be

$$
R_{l}^{*}=\frac{R\left[\left(4 \gamma_{l}^{2} \pi^{2}\right)+\left(\gamma_{b}-\gamma_{s}-\gamma_{l b}\right)^{2}\right]}{4 \gamma_{l}^{2} \pi^{2}} .
$$

where $\gamma l$ is the free surface energy of the liquid-vapor interface and is the interfacial energy between the liquid and substrate.

\section{References}

[1] P. Pawlow, "The dependency of the melting point on the surface energy of a solid body," Zeitschrift für Physikalische Chemie, vol. 65, no. 5, pp. 545-548, 1909.

[2] D. K. Sar, P. Nayak, and K. K. Nanda, “Thermodynamic model for the size-dependent melting of prism-shaped nanoparticles," Physics Letters A, vol. 372, no. 25, pp. 4627-4629, 2008.
[3] I. Gulseren and J. N. Coupland, "Surface melting in alkane emulsion droplets as affected by surfactant type," Journal of the American Oil Chemists' Society, vol. 85, no. 5, pp. 413-419, 2008.

[4] W. H. Qi, "Size effect on melting temperature of nanosolids," Physica B, vol. 368, no. 1-4, pp. 46-50, 2005.

[5] G. Guisbiers and M. Wautelet, "Size, shape and stress effects on the melting temperature of nano-polyhedral grains on a substrate," Nanotechnology, vol. 17, no. 8, pp. 2008-2011, 2006.

[6] A. S. Shirinyan, A. M. Gusak, and M. Wautelet, "Melting and superheating of nanowires," Acta Materialia, vol. 55, article 5025, 2005.

[7] G. Guisbiers, O. van Overschelde, and M. Wautelet, "Nanoparticulate orgin of intrinsic residual strees in thin films," Acta Materialia, vol. 55, article 3541, 2007.

[8] C. Q. Sun, Y. Wang, B. K. Tay, S. Li, H. Huang, and Y. B. Zhang, "Correlation between the melting point of a nanosolid and the cohesive energy of a surface atom," Journal of Physical Chemistry B, vol. 106, no. 41, pp. 10701-10705, 2002.

[9] C. Kan, G. Wang, X. Zhu, C. Li, and B. Cao, "Structural and thermal stability of gold nanoparticle," Applied Physics Letters, vol. 88, Article ID 071904, 2006.

[10] H. H. Farrell, "Surface bonding effects in compound semiconductor nanoparticles: II," Journal of Vacuum Science and Technology B, vol. 26, no. 4, pp. 1534-1541, 2008.

[11] A. Jiang, N. Awasthi, A. N. Kolmogorov et al., "Theoretical study of the thermal behavior of free and alumina-supported Fe-C nanoparticles," Physical Review B, vol. 75, no. 20, Article ID 205426, 2007.

[12] P. Pawlow, "Size dependent melting properties of Bi nanoparticles by molecular dynamics simulation," Physical Chemistry, vol. 74, article 562, 1910.

[13] T. Bachels, H. J. Guntherodt, and R. Schafer, "Melting of isolated tin nanoparticles," Physical Review Letters, vol. 85, no. 6, pp. 1250-1253, 2000.

[14] E. A. Olsan, M.Yu. Efermov, M. Zhang, Z. Zhang, and L. H. Allen, "Size-dependent melting of Bi nanoparticles," Journal of Applied Physics, vol. 97, no. 3, Article ID 034304, 9 pages, 2005.

[15] M. Zhang, M. Y. Efremov, F. Schiettekatte et al., "Sizedependent melting point depression of nanostructures: nanocalorimetric measurements," Physical Review B, vol. 62, no. 15, pp. 10548-10557, 2000.

[16] M. D. McCluckey, Y. M. Gupta, C. G. van de Walle, D. P. Bour, M. Kneissl, and N. M. Johnson, "Band gap changes of GaN shacked to BGpa," Applied Physics Letters, vol. 80, pp. 19121914, 2002.

[17] X. W. Wang, G. T. Fei, K. Zheng, Z. Jin, and L. de Zhang, "Sizedependent melting behavior of Zn nanowire arrays," Applied Physics Letters, vol. 88, no. 17, Article ID 173114, 2006.

[18] G. Mula, C. Adelmann, S. Moehl, J. Oullier, and B. Daudin, "Surfactant effect of gallium during molecular-beam epitaxy of GaN on AIN (0001)," Physical Review B, vol. 64, no. 19, Article ID 195406, pp. 1954061-19540612, 2001.

[19] B. Heying, R. Averbeck, L. F. Chen, E. Haus, H. Riechert, and J. S. Speck, "Control of GaN surface morphologies using plasma-assisted molecular beam epitaxy," Journal of Applied Physics, vol. 88, no. 4, pp. 1855-1860, 2000.

[20] L. X. Zheng, M. H. Xie, S. M. Seutter, S. H. Cheung, and S. Y. Tong, "Observation of "ghost" islands and surfactant effect of surface gallium atoms during GaN growth by molecular beam epitaxy," Physical Review Letters, vol. 85, no. 11, pp. 23522355, 2000. 
[21] C. Adelmann, J. Brault, G. Mula, B. Daudin, L. Lymperakis, and J. Neugebauer, "Gallium adsorption on (0001) GaN surfaces," Physical Review B, vol. 67, no. 16, Article ID 165419, pp. 1654191-1654199, 2003.

[22] G. Koblmuller, R. Averbeck, H. Riechert, and P. Pongratz, "Direct observation of different equilibrium Ga adlayer coverages and their desorption kinetics on GaN (0001) and (0001) surfaces," Physical Review B, vol. 69, no. 3, Article ID 035325, pp. 353251-353259, 2004.

[23] P. H. Buffat and J. P. Borel, "Size effect on the melting temperature of gold particles," Physical Review A, vol. 13, no. 6, pp. 2287-2298, 1976.

[24] P. R. Couchman and W. A. Jesser, "Thermodynamic theory of size dependence of melting temperature in metals," Nature, vol. 269, no. 5628, pp. 481-483, 1977.

[25] T. Castro, R. Reifenberger, E. Choi, and R. P. Andres, "Sizedependent melting temperature of individual nanometersized metallic clusters," Physical Review B, vol. 42, no. 13, pp. 8548-8556, 1990.

[26] K. F. Peters, J. B. Cohen, and Y. W. Chung, "Melting of Pb nanocrystals," Physical Review B, vol. 57, no. 21, pp. 1343013438, 1998.

[27] M. Zhao, X. H. Zhou, and Q. Jiang, "Comparison of different models for melting point change of metallic nanocrystals," Journal of Materials Research, vol. 16, no. 11, pp. 3304-3307, 2001.

[28] K. Dick, T. Dhanasekaran, Z. Zhang, and D. Meisel, "Sizedependent melting of silica-encapsulated gold nanoparticles," Journal of the American Chemical Society, vol. 124, no. 10, pp. 2312-2317, 2002.

[29] K. K. Nanda and S. C. Vanithakumari, "A universal relation for the cohesive energy of nanoparticles," Physics Letters A, vol. 372, no. 46, pp. 6930-6934, 2008.

[30] C. Q. Sun, Y. Wang, B. K. Tay, S. Li, H. Huang, and Y. B. Zhang, "Correlation between the melting point a nanoslid and the cohersive energy of a surface atom," Journal of Physical Chemistry B, vol. 106, no. 41, pp. 10701-10705, 2002.

[31] W. A. Jesser, R. Z. Shneck, and W. W. Gile, "Solid-liquid equilibria in nanoparticles of $\mathrm{Pb}-\mathrm{Bi}$ alloys," Physical Review B, vol. 69, no. 14, Article ID 144121, 13 pages, 2004.

[32] S. Cuenot, C. Fretigny, S. D. Champagne, and B. Nysten, "Surface tension effect on the mechanical properties of nanomaterials measured by atomic force microscopy," Physical Review B, vol. 69, no. 16, Article ID 165410, 5 pages, 2004.

[33] K. Kendall, "The impossibility of comminuting small particles by compression," Nature, vol. 272, no. 5655, pp. 710-711, 1978.

[34] B. L. Karihaloo, "Scaling laws for properties of materials with imperfect interfaces," Nature, vol. 279, articel 169, 1979.

[35] P. Antoniammal and D. Arivuoli, "A thermo dynamical model for the shape and size effect on melting of boron carbide nanoparticles," Journal of Nanoscience and Nanotechnology, vol. 11, pp. 1-8, 2011.

[36] S. C. Hendy, "A thermodynamic model for the melting of supported metal nanoparticles," Nanotechnology, vol. 18, no. 17, Article ID 175703, 2007.

[37] C. R. M. Wronski, “The size dependence of the melting point of small particles of tin," British Journal of Applied Physics, vol. 18, no. 12, pp. 1731-1737, 1967.

[38] D. Kashchiev and G. M. van Rosmalen, "Nucleation in solutions revisited," Crystal Research and Technology, vol. 38, no. 7-8, pp. 555-574, 2003.
[39] F. Ercolessi, W. Andreoni, and E. Tosatti, "Melting of small gold particles: Mechanism and size effects," Physical Review Letters, vol. 66, no. 7, pp. 911-914, 1991.

[40] L. J. P. Lewis, P. Jensen, and J. L. Barrat, "Melting, freezing, and coalescence of gold nanoclusters," Physical Review B, vol. 56, no. 4, pp. 2248-2257, 1997.

[41] G. L. Allen, R. A. Bayles, W. W. Gile, and W. A. Jesser, "Small particle melting of pure metals," Thin Solid Films, vol. 144, no. 2, pp. 297-308, 1986.

[42] J. Sun and S. L. Simon, "The melting behavior of aluminum nanoparticles," Thermochimica Acta, vol. 463, no. 1-2, pp. 3240, 2007.

[43] F. Lopeandia and J. Rodriguez-Viejo, "Size-dependent melting and supercooling of $\mathrm{Ge}$ nanoparticles embedded in $\mathrm{a} \mathrm{SiO}_{2}$ thin film," Thermochimica Acta, vol. 461, no. 1-2, pp. 82-87, 2007.

[44] M. Takagi, "Electron-diffraction study of liquid-solid transition of thin metal films," Journal of the Physical Society of Japan, vol. 9, no. 3, pp. 359-363, 1954.

[45] W. H. Qi and M. P. Wang, "Size effect on the cohesive energy of nanoparticle," Journal of Materials Science Letters, vol. 21, no. 22, pp. 1743-1745, 2002.

[46] K. K. Nanda, S. N. Sahu, and S. N. Behera, "Liquid-drop model for the size-dependent melting of low-dimensional systems," Physical Review A, vol. 66, no. 1, Article ID 013208, 2002.

[47] J. W. M. Frenken and J. F. van der Veen, "Observation of surface melting," Physical Review Letters, vol. 54, no. 2, pp. 134-137, 1985.

[48] H. Sakai, "Surface-induced melting of small particles," Surface Science, vol. 351, no. 1-3, pp. 285-291, 1996.

[49] C. Q. Sun, Y. Wang, B. K. Tay, S. Li, H. Huang, and Y. B. Zhang, "Correlation between the melting point of a nanosolid and the cohesive energy of a surface atom," Journal of Physical Chemistry B, vol. 106, no. 41, pp. 10701-10705, 2002.

[50] H. K. Kim, S. H. Huh, J. W. Park, J. W. Jeong, and G. H. Lee, "Modeling the cohersive energy and melting point of nanoparticles," Chemical Physics Letters, vol. 354, article 165, 2002.

[51] W. H. Qi and M. P. Wang, "A simplified model to calculate the surface-to-volume atomic ratio dependent cohersive energy of nanocrystals," Journal of Materials Science Letters, vol. 21, article 1743, 2002.

[52] W. H. Qi, M. P. Wang, and G. Y. Xu, "An investigation of the size-dependent cohersive energy and the structural stability of spherical metallic nanoparticles," Chemical Physics Letters, vol. 372, article 632, 2003.

[53] W. H. Qi, M. P. Wang, and W. Y. Hu, "Nano structured materials for advanced technological applications," Materials Letters, vol. 58, article 1745, 2004.

[54] Q. Jiang, J. C. Li, and B. Q. Chi, "Size-dependent cohesive energy of nanocrystals," Chemical Physics Letters, vol. 366, no. 5-6, pp. 551-554, 2002.

[55] K. K. Nanda, F. E. Kruis, and H. Fissan, "Evaporation of free PbN nanoparticles: evidence of the Kelvin effect," Physical Review Letters, vol. 89, no. 25, Article ID 256103, 4 pages, 2002.

[56] C. Brechignac, H. Busch, P. H. Cahuzac, and J. Leygnier, "Dissociation pathways and binding energies of lithium clusters from evaporation experiments," The Journal of Chemical Physics, vol. 101, no. 8, pp. 6992-7002, 1994.

[57] F. Chandezon, P. M. Hansen, C. Ristori, J. Pedersen, J. Westergaard, and S. Bjørnholm, "Evaporation of clusters in a heat bath," Chemical Physics Letters, vol. 277, no. 5-6, pp. 450-455, 1997. 
[58] S. L. Lai, J. Y. Guo, V. Petrova, G. Ramanath, and L. H. Allen, "Size-dependent melting properties of small tin particles: nanocalorimetric measurements," Physical Review Letters, vol. 77, no. 1, pp. 99-102, 1996.

[59] C. Q. Sun, B. K. Tay, X. T. Zeng et al., "A nanosolid and the cohersive energy of a surface atoms," Journal of Physics, vol. 14, article 7781, 2002.

[60] D. Xie, W. H. Qi, L. F. Cao, and M. P. Wang, "Thermal stability of indium nanocrystals: a theoretical study," Materials Chemistry and Physics, vol. 96, no. 2-3, pp. 418-421, 2006.

[61] Z. Wang, X. Zu, F. Gao, and W. J. Weber, "Size dependence of melting of GaN nanowires with triangular cross sections," Journal of Applied Physics, vol. 101, no. 4, Article ID 043511, 2007.

[62] K. Rollins, "Nanobiotechnology regulation: a proposal for selfregulation with limited oversight," Nems Mems Works, LLC, vol. 6, no. 2, 2010.

[63] G. Guisbiers, D. Liu, Q. Jiang, and L. Buchaillot, "Theoretical predictions of wurtzite III-nitride nano-materials properties," Physical Chemistry Chemical Physics, vol. 12, no. 26, pp. 72037210, 2010.

[64] Y. H. Wen, Z. Zhu, R. Zhu, and G. F. Shao, "Size-dependent melting of nanoparticles," Physica E, vol. 25, article 47, 2004. 

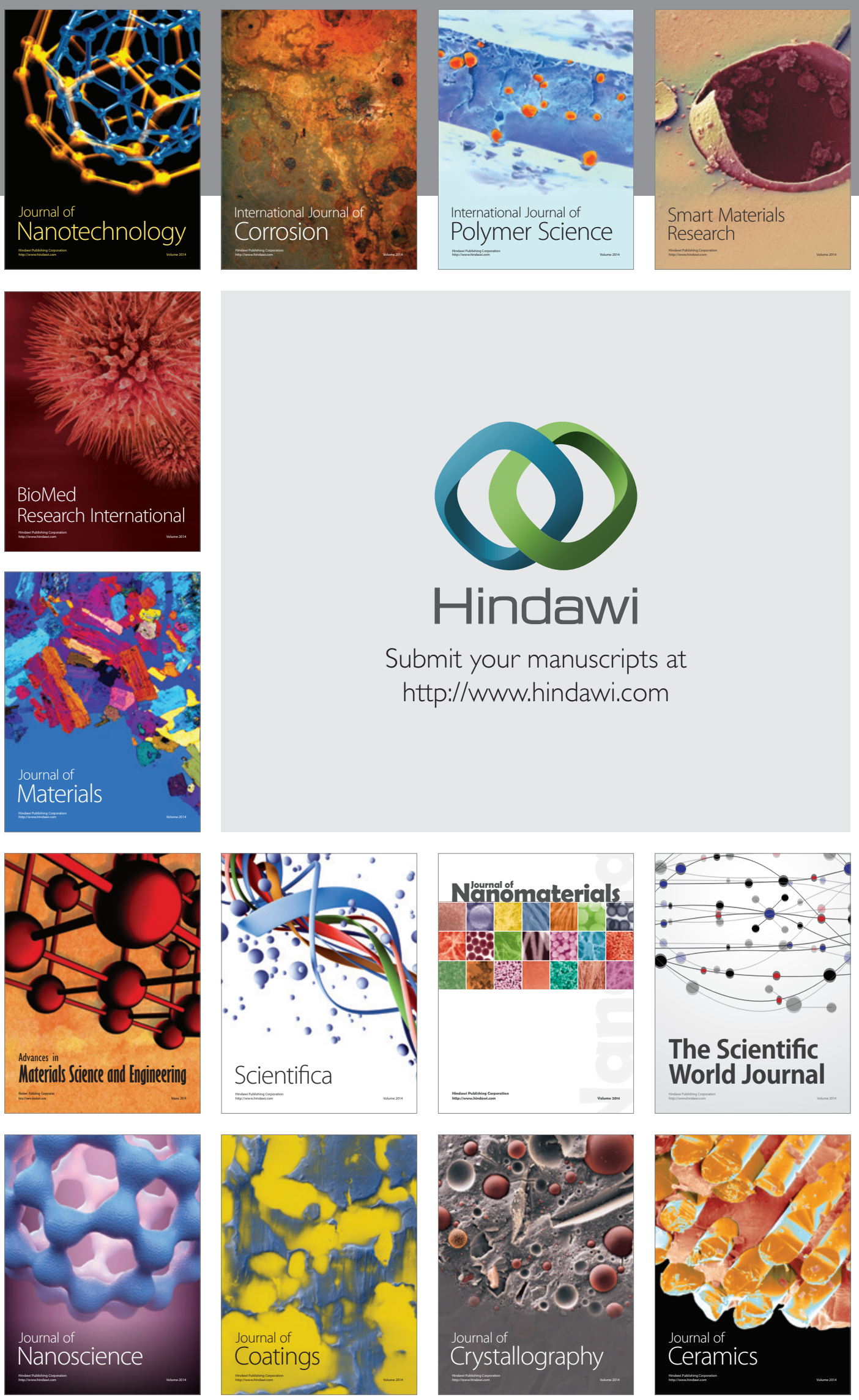

The Scientific World Journal

Submit your manuscripts at

http://www.hindawi.com

\section{World Journal}

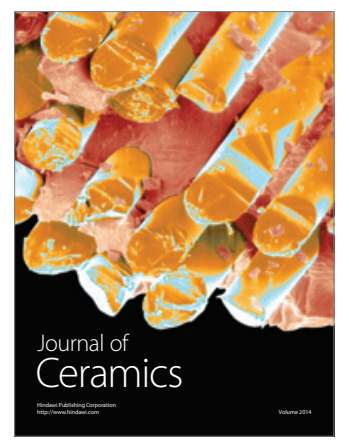

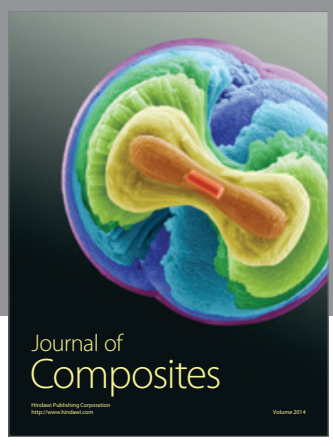
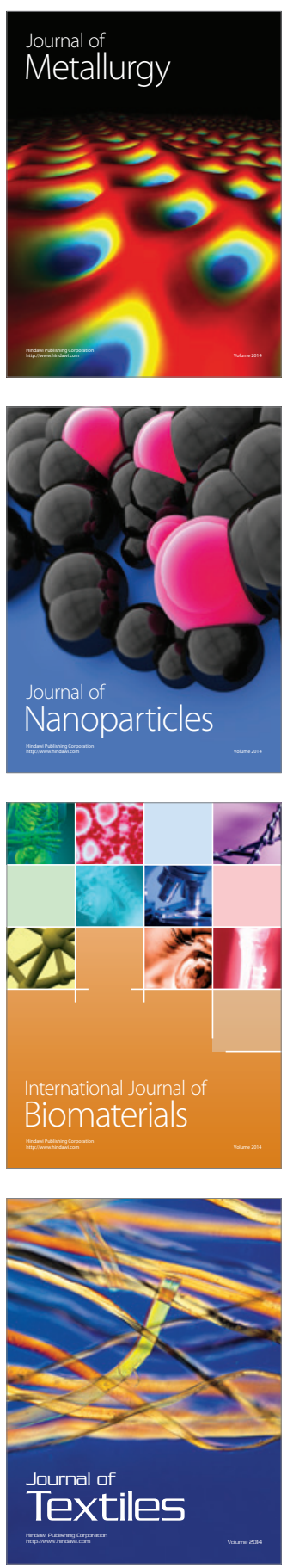